\title{
A MENŠOV TYPE THEOREM
}

\author{
T. W. KÖRNER \\ DPMMS, 16 Mill Lane, Cambridge, U.K. \\ E-mail: twk@pmms.cam.ac.uk
}

\begin{abstract}
Any integrable function can be corrected, so that its Fourier series converges almost everywhere to the correct limit, by the addition of a measure whose singular support is small both in the algebraic and the Hausdorff sense.
\end{abstract}

1. Introduction. I should like to thank the organisers for a conference which was extraordinarily enjoyable for many reasons - one of which was the opportunity to meet mathematicians whose work I have long admired but whom I had never met. My talk at the conference was based on [1], but this paper contains new results on the topic suggested to me by conversations at the conference.

In this paper we identify $\mathbb{T}$ with $[0,2 \pi)$ in the usual way. All measures will be Borel measures and, where we do not specify otherwise, the Haar measure (that is normalised Lebesgue measure) $m$ is intended. If $E$ is measurable, we write $|E|$ for the measure $m(E)$ of $E$. If $f \in L^{1}(\mathbb{T})$, we write

$$
S_{n}(f, x)=\sum_{|r| \leq n} \hat{f}(r) \exp i r x .
$$

If $\mu$ is a measure, $S_{n}(\mu, x)$ is defined similarly. We write $A(\mathbb{T})$ for the set of absolutely convergent Fourier series and, if $f \in A(\mathbb{T})$, we write $\|f\|_{A}=\sum_{u=-\infty}^{\infty}|\hat{f}(u)|$. If $\mu$ is a measure then supp $\mu$ denotes its closed support.

In [1] I proved the following result which is closely related to various results of Menšov.

THEOREM 1. Given any $f \in L^{1}$, and any $\epsilon>0$, there exists a singular measure $\mu$ with $\|\mu\| \leq \epsilon$ such that

$$
S_{n}(f-\mu, x) \rightarrow f(x)
$$

almost everywhere as $n \rightarrow \infty$.

2000 Mathematics Subject Classification: Primary 42A20; Secondary 42A63.

Key words and phrases: trigonometric series, adjustment of functions.

The paper is in final form and no version of it will be published elsewhere. 
It is natural to ask 'how small' the measure $\mu$ must be. Here is one answer to this question.

THEOREM 2. Given any $f \in L^{1}$ any strictly increasing continuous $h:[0, \infty) \rightarrow[0, \infty)$ with $h(0)=0$ and any $\epsilon>0$, there exists a measure $\mu$ with $\|\mu\| \leq \epsilon$ and an independent Borel set $E$ with zero Hausdorff $h$ measure such that $|\mu|(\mathbb{T} \backslash E)=0$ and

$$
S_{n}(f-\mu, x) \rightarrow f(x)
$$

almost everywhere as $n \rightarrow \infty$.

The reader may wish to be reminded of the following definitions.

Definition 3. (i) A set $E$ is independent if, whenever $n \geq 1$ and $x_{1}, x_{2}, \ldots, x_{n}$ are distinct points in $E$, the only solution of the equation

$$
\sum_{j=1}^{n} m_{j} x_{j}=0
$$

with the $m_{j}$ integers is $m_{1}=m_{2}=\ldots=m_{n}=0$.

(ii) If $h:[0, \infty) \rightarrow[0, \infty)$ is a strictly increasing continuous function with $h(0)=0$, then a set $E$ has zero Hausdorff $h$ measure if, given any $\epsilon>0$, we can find intervals $I_{1}$, $I_{2}, \ldots$ such that $\bigcup_{j=1}^{\infty} I_{j} \supseteq E$ and $\sum_{j=1}^{\infty} h\left(\left|I_{j}\right|\right)<\epsilon$.

The main ideas of the construction appear in the proof of the simpler result.

THEOREM 4. Given any $f \in L^{1}$ any strictly increasing continuous $h:[0, \infty) \rightarrow[0, \infty)$ with $h(0)=0$ and any $\epsilon>0$, there exists a measure $\mu$ with $\|\mu\| \leq \epsilon$ and a Borel set $E$ of zero Hausdorff $h$ measure such that $|\mu|(\mathbb{T} \backslash E)=0$ and

$$
S_{n}(f-\mu, x) \rightarrow f(x)
$$

almost everywhere as $n \rightarrow \infty$.

We shall therefore concentrate first on proving this result and sketch the more intricate proof towards the end of the paper.

2. The key lemma. The main step in the proof of Theorem 4 is the proof of the following lemma.

Lemma 5. Given any $\eta>0$ we can find $a K_{1}(\eta)>1$ with the following property. Given any strictly increasing continuous function $h:[0, \infty) \rightarrow[0, \infty)$ with $h(0)=0$ and any $\epsilon>0$ we can find a positive measure $\mu$ and a Borel set $G$ with

(i) $\hat{\mu}(0)=1$,

(ii) $\hat{\mu}(n) \rightarrow 0$ as $|n| \rightarrow \infty$,

(iii) $|\hat{\mu}(u)| \leq \epsilon$ for all $u \neq 0$,

(iv) $\left|S_{n}(\mu, x)\right| \leq K_{1}(\eta)$ for all $n \geq 0$ and all $x \notin G$,

(v) $|G|<\eta$,

(vi) $\operatorname{supp} \mu$ has Hausdorff $h$ measure zero.

In this section we show how to prove Theorem 4 using Lemma 5, and, in the next section, we prove Lemma 5. 
Lemma 6. Given any $\eta>0$ we can find a $K_{2}(\eta)>1$ with the following property. Given any trigonometric polynomial $Q$ and any strictly increasing continuous function $h:[0, \infty) \rightarrow[0, \infty)$ with $h(0)=0$ and any $\epsilon>0$, we can find a measure $\tau$ and a Borel set $G$ with

(i) $\|\tau\| \leq\|Q\|_{1}$,

(ii) $\hat{\tau}(n) \rightarrow 0$ as $|n| \rightarrow \infty$,

(iii) $\left|S_{n}(\tau, x)-S_{n}(Q, x)\right| \leq K_{2}(\eta)|Q(x)|$ for all $n \geq 0$ and all $x \notin G$,

(iv) $|G|<\eta$,

(v) $\operatorname{supp} \tau$ has Hausdorff $h$ measure zero.

Proof. Suppose that, for each $\epsilon>0$ we have a positive measure $\mu_{\epsilon}$ such that

(i) $\hat{\mu}_{\epsilon}(0)=1$,

(ii) $\hat{\mu}_{\epsilon}(n) \rightarrow 0$ as $|n| \rightarrow \infty$,

(iii) $\left|\hat{\mu}_{\epsilon}(u)\right| \leq \epsilon$ for all $u \neq 0$.

If we set $\tau_{\epsilon}=Q \mu_{\epsilon}$ then $\left\|\tau_{\epsilon}\right\| \leq\|Q\|_{\infty}$ and

$$
\int_{\mathbb{T}} P(t) d \tau_{\epsilon}(t)=\int_{\mathbb{T}} P(t) Q(t) d \mu_{\epsilon}(t) \rightarrow \int_{\mathbb{T}} P(t) Q(t) d m(t)
$$

as $\epsilon \rightarrow 0$, so a simple density argument shows that

$$
\left\|\tau_{\epsilon}\right\| \rightarrow\|Q\|_{1}
$$

as $\epsilon \rightarrow 0$.

Next we observe that

$$
\left|\mu_{\epsilon} \widehat{-m}(u)\right| \leq \epsilon \text { for all } u
$$

and so

$$
\left(S_{n}\left(\tau_{\epsilon}, t\right)-S_{n}(Q, t)\right)-Q(t) S_{n}\left(\mu_{\epsilon}, t\right)=S_{n}\left(Q\left(\mu_{\epsilon}-m\right), t\right)-Q(t) S_{n}\left(\mu_{\epsilon}, t\right) \rightarrow 0
$$

uniformly in $t$ and $n \geq 0$ as $\epsilon \rightarrow 0$ (Similar calculations are done at length in [1].)

Thus, if we set $K_{2}(\eta)=K_{1}(\eta)+1$, take $\epsilon$ sufficiently small, take $\mu$ as in the conclusion of Lemma 6 and set

$$
\tau=k Q \mu
$$

with $k$ chosen so that $\|\tau\|=\|Q\|_{1}$, all the conclusions required can be read off.

We can now prove Theorem 4.

Proof of Theorem 4. Let $K_{2}$ be defined as in the statement of Lemma 6. We may assume $1>\epsilon$ and we set $\epsilon_{j}=2^{-2 j} \epsilon K_{2}\left(2^{-j}\right)^{-1}$. If $f \in L^{1}$ we can find trigonometric polynomials $Q_{j}$ such that $\left\|Q_{j}\right\|_{1}<\epsilon_{j}$ for $j \geq 1$ and $\sum_{j=0}^{\infty} Q_{j}=f$, the convergence being both $L^{1}$ and pointwise almost everywhere. In particular we can find a set $B$ of measure zero such that $\sum_{j=0}^{N} Q_{j}(x) \rightarrow f(x)$ for all $x \notin B$.

By Lemma 6 we can find measures $\tau_{j}$ and a closed set $G_{j}$ with

(i) $)_{j}\left\|\tau_{j}\right\| \leq\left\|Q_{j}\right\|_{1}$,

(ii) ${ }_{j} \hat{\tau}_{j}(n) \rightarrow 0$ as $|n| \rightarrow \infty$,

(iii) ${ }_{j}\left|S_{n}\left(\tau_{j}, x\right)-S_{n}\left(Q_{j}, x\right)\right| \leq K_{2}\left(2^{-j}\right)\left|Q_{j}(x)\right|$ for all $n \geq 0$ and all $x \notin G_{j}$, 
(iv) ${ }_{j}\left|G_{j}\right|<2^{-j}$

$(\mathrm{v})_{j} \operatorname{supp} \tau_{j}$ has Hausdorff $h$ measure zero.

Since $\left\|\tau_{j}\right\| \leq\left\|Q_{j}\right\|_{1}<\epsilon_{j} \leq \epsilon 2^{-j}$, we can form the measure $\mu=\sum_{j=1}^{\infty} \tau_{j}$ with $\|\mu\| \leq \epsilon$. If we set $E=\bigcup_{j=1}^{\infty} \operatorname{supp} \tau_{j}$ then $E$ is a Borel set such that $|\mu|(\mathbb{T} \backslash E)=0$ and, by condition (v), $E$ has zero Hausdorff $h$ measure.

Since $\left\|Q_{j}\right\|_{1}<\epsilon_{j} \leq 2^{-2 j} K_{2}\left(2^{-j}\right)^{-1}$, a simple Tchebychev type estimate shows that, if

$$
H_{j}=\left\{x:\left|Q_{j}(x)\right| \geq 2^{-j} K_{2}\left(2^{-j}\right)^{-1}\right\},
$$

then $\left|H_{j}\right| \leq 2^{-j}$. Thus, setting $A_{j}=G_{j} \cup H_{j}$ and using (iii) ${ }_{j}$, and (iv) ${ }_{j}$ we have

(vi) ${ }_{j}\left|S_{n}\left(\tau_{j}, x\right)-S_{n}\left(Q_{j}, x\right)\right| \leq 2^{-j}$ for all $n \geq 0$ and all $x \notin A_{j}$,

(vii) $)_{j}\left|A_{j}\right|<2^{-j+1}$.

Thus if we set

$$
A=B \cup \bigcup_{j=1}^{\infty} \operatorname{supp} \tau_{j} \cup \bigcap_{j=1}^{\infty} \bigcup_{k=j}^{\infty} A_{j},
$$

it follows that $A$ has Lebesgue measure zero. We complete the proof by showing that

$$
S_{n}(f-\mu, x) \rightarrow f(x)
$$

as $n \rightarrow \infty$ for all $x \in A$.

By classical localisation theorems,

$$
S_{n}\left(\tau_{j}, x\right) \rightarrow 0
$$

for all $x \notin \operatorname{supp} \tau_{j}$, and, since $Q_{j}$ is a trigonometric polynomial, it is trivial that

$$
S_{n}\left(Q_{j}, x\right) \rightarrow Q_{j}(x)
$$

for all $x$. Thus if

$$
x \notin B \cup \bigcup_{j=1}^{N} \operatorname{supp} \tau_{j} \cup \bigcup_{k=N+1}^{\infty} A_{j}
$$

we have

$$
\begin{aligned}
\left|S_{n}(f-\mu, x)-f(x)\right| \leq & \sum_{j=1}^{N}\left|S_{n}\left(\tau_{j}, x\right)\right|+\sum_{j=1}^{N}\left|S_{n}\left(Q_{j}, x\right)-Q_{j}(x)\right| \\
& +\sum_{j=N+1}^{\infty}\left|S_{n}\left(\tau_{j}, x\right)-S_{n}\left(Q_{j}, x\right)\right|+\sum_{j=N+1}^{\infty}\left|Q_{j}(x)\right| \\
\leq & \sum_{j=1}^{N}\left|S_{n}\left(\tau_{j}, x\right)\right|+\sum_{j=1}^{N}\left|S_{n}\left(Q_{j}, x\right)-Q_{j}(x)\right| \\
& +\sum_{j=N+1}^{\infty} 2^{-j}+\sum_{j=N+1}^{\infty} 2^{-j} \\
\leq & \sum_{j=1}^{N}\left|S_{n}\left(\tau_{j}, x\right)\right|+\sum_{j=1}^{N}\left|S_{n}\left(Q_{j}, x\right)-Q_{j}(x)\right|+2^{-N+1}
\end{aligned}
$$


and so

$$
\limsup _{n \rightarrow \infty}\left|S_{n}(f-\mu, x)-f(x)\right| \leq 2^{-N+1} .
$$

Allowing $N \rightarrow \infty$, we have the result.

3. Proof of the key lemma. The measure $\mu$ in our key Lemma 5 is obtained as the weak star limit of a sequence of smooth functions.

LEMMA 7. Given any $\eta>0$ we can find $a K_{1}(\eta)>1$ with the following property. Given any strictly increasing continuous function $h:[0, \infty) \rightarrow[0, \infty)$ with $h(0)=0$ and any $\epsilon>0$ we can find a sequence of positive infinitely differentiable functions $f_{k}$ and sets $G_{k}$ such that

$(i)_{k} \hat{f}_{k}(0)=1$,

(ii) $)_{k}\left|\hat{f}_{k}(u)-\hat{f}_{k-1}(u)\right| \leq 2^{-k}$ for all $u$ if $k \geq 2$,

$(\text { iii })_{k}\left|\hat{f}_{k}(u)\right| \leq \epsilon\left(1-2^{-k}\right)$ for all $u \neq 0$,

$(\text { iv })_{k}\left|S_{n}\left(f_{k}, x\right)\right| \leq K_{1}(\eta)\left(1-2^{-k}\right)$ for all $n \geq 0$ and all $x \notin G_{k}$,

$(v)_{k}\left|G_{k}\right|<\eta\left(1-2^{-k)}\right)$,

$(\text { vi })_{k}$ If $k \geq 2$ we can find a finite set $\mathcal{I}(k)$ of closed intervals such that $\bigcup_{I \in \mathcal{I}(k)} I \supseteq$ $\operatorname{supp} f_{k}$ but $\sum_{I \in \mathcal{I}(k)} h(|I|) \leq 2^{-k}$,

(vii) ${ }_{k} G_{k} \supseteq G_{k-1}$ if $k \geq 2$,

(viii) $)_{k} \operatorname{supp} f_{k-1} \supseteq \operatorname{supp} f_{k}$ if $k \geq 2$,

$(i x)_{k} G_{k} \supseteq \operatorname{supp} f_{k}$.

Proof of Lemma 5 from Lemma \%. Using conditions (i) ${ }_{k}$ and (ii) $)_{k}$, we see that $f_{k} m$ converges weakly to a positive measure $\mu$ with

(i) $\hat{\mu}(0)=1$.

Since $f_{n}$ is smooth $\hat{f}_{k}(u) \rightarrow 0$ as $n \rightarrow \infty$ for each $k$ so

(ii) $\hat{\mu}(n) \rightarrow 0$ as $|n| \rightarrow \infty$.

Condition $(\mathrm{iii})_{k}$ shows that

(iii) $|\hat{\mu}(u)| \leq \epsilon$ for all $u \neq 0$,

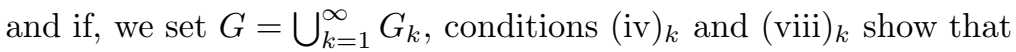

(iv) $\left|S_{n}(\mu, x)\right| \leq K_{1}(\eta)$ for all $n \geq 0$ and all $x \notin G$,

whilst conditions $(\mathrm{v})_{k}$ and (vii) $k$ show that

(v) $|G|<\eta$.

Finally, conditions (viii) $k$ and $(\mathrm{vi})_{k}$ show that

(vi) $\operatorname{supp} \mu$ has Hausdorff $h$ measure zero.

It is important to observe that the first step in proving Lemma 7 is of a different nature to the others and, so far as I can see, non-trivial. To see why this might be the case observe that whilst condition $(\mathrm{vi})_{k}$ shows an improvement as we increase $k$, this improvement is bought at the cost of a deterioration in conditions (iv) $k,(\mathrm{v})_{k}$ and (iii) . $_{\text {. }}$ 
Looking more closely, we see that our first step requires us to prove the following lemma.

LEMMA 8. Given any $\eta>0$ we can find a $K_{1}(\eta)>1$ with the following property. Given any $\epsilon>0$ we can find a positive infinitely differentiable function $f_{1}$ and a set $G_{1}$ such that

(i) $\hat{f}_{1}(0)=1$,

(iii) $1\left|\hat{f}_{1}(u)\right| \leq \epsilon / 2$ for all $u \neq 0$,

(iv) $\left|S_{n}\left(f_{1}, x\right)\right| \leq K_{1}(\eta) / 2$ for all $n \geq 0$ and all $x \notin G_{1}$,

$(v)_{1}\left|G_{1}\right|<\eta / 2$,

(vii) ${ }_{1} G_{1}$ is the union of a finite set of closed intervals with end points rational multiples of $2 \pi$,

$(x)_{1} G_{1} \supseteq \operatorname{supp} f_{1}$.

The difficult point here is that we may choose $\epsilon$ as small as we like without affecting $K_{1}(\eta)$. The result is proved as Lemma 2 in [1].

4. The inductive step for the key lemma. We observe that the induction required for Lemma 7 and thus the result required will be established if we can prove the following result.

Lemma 9. Let $h:[0, \infty) \rightarrow[0, \infty)$ be a strictly increasing continuous function with $h(0)=0$. Suppose that $f$ is a positive infinitely differentiable function with $\hat{f}(0)=1$. Then, given any $\delta>0$, we can find a positive infinitely differentiable function $F$ and a closed set $H$ such that

(i) $\hat{F}(0)=1$,

(ii) $|\hat{F}(u)-\hat{f}(u)| \leq \delta$,

(iii) $\left|S_{n}(F, x)\right| \leq\left|S_{n}(f, x)\right|+\delta$ for all $n \geq 0$ and all $x \notin H$,

(iv) We can find a finite set $\mathcal{I}$ of closed intervals such that $\bigcup_{I \in \mathcal{I}} I \supseteq \operatorname{supp} F$ but $\sum_{I \in \mathcal{I}} h(|I|) \leq \delta$

(v) $\operatorname{supp} F \subseteq \operatorname{supp} f$,

(vi) $H \supseteq \operatorname{supp} f$,

(vii) $|H|<|\operatorname{supp} f|+\delta$.

Justification of the inductive step in Lemma \%. Set $f_{k}=f, f_{k+1}=F$ and $G_{k+1}=$ $G_{k} \cup H$. Provided that $\delta$ is small enough, we have the desired result. Note that we need to know that $H \supseteq \operatorname{supp} f_{k}$ and $G_{k} \supseteq \operatorname{supp} f_{k}$ in order to obtain (v) $k+1$ from (vii) and (v) $)_{k}$.

However, things are not quite as simple as a hasty inspection of Lemma 9 might lead one to believe. The inductive step itself needs (so far as I can see) to be broken up into several steps and (again so far as I can see) it is here that we need to be most careful.

We start with a preparatory step.

LEMMA 10. Suppose that $f$ is a positive infinitely differentiable function with $\hat{f}(0)$ $=1$. Then, given any $\delta>0$, we can find an $\eta>0$, a finite collection $\mathcal{K}$ of closed intervals such that every pair of intervals in $\mathcal{K}$ intersect at at most one point, and a closed set $H$ such that 
(a) $H \supseteq \operatorname{supp} f$,

(b) $|H|<|\operatorname{supp} f|+\delta$,

(c) If $x \notin H$ and $y \in \bigcup_{K \in \mathcal{K}} K$ then $|x-y|>\eta$,

(d) If $K \in \mathcal{K}$ then $10^{2} \eta^{-1} \int_{K} f(t) d t<\delta$.

Proof. Since supp $f$ is closed we can find a finite collection of intervals $J_{1}, J_{2}, \ldots, J_{m}$, say with $\bigcup_{r=1}^{m} J_{r} \supseteq \operatorname{supp} f$ and $\sum_{r=1}^{m}\left|J_{r}\right|<|\operatorname{supp} f|+\delta / 2$. Choose $\eta>0$ such that $8 m \eta<\delta$ and set

$$
H=\bigcup_{r=1}^{m}\left(J_{r}+[-2 \eta, 2 \eta]\right)
$$

where we write $[a, b]+[-2 \eta, 2 \eta]=[a-2 \eta, b+2 \eta]$. Conditions (a) and (b) are automatic.

Now choose $\mathcal{K}$ to be a finite collection of closed intervals such that

(i) $\bigcup_{K \in \mathcal{K}} K=\bigcup_{r=1}^{m} J_{r}$,

(ii) every pair of intervals in $\mathcal{K}$ intersect at at most one point,

(iii) if $K \in \mathcal{K}$ then $|K|<\|f\|_{\infty}^{-1} 10^{-2} \eta \delta$.

Conditions (c) and (d) are automatic.

We now need to apply the following lemma repeatedly.

Lemma 11. Let $h:[0, \infty) \rightarrow[0, \infty)$ be a strictly increasing continuous function with $h(0)=0$. Suppose that we are given $f$ a positive infinitely differentiable function with $\hat{f}(0)=1, \delta>0$ and $\eta>0$, together with a finite collection $\mathcal{K}$ of closed intervals such that every pair of intervals in $\mathcal{K}$ intersect at at most one point, and a closed set $H$ such that

(a) $H \supseteq \operatorname{supp} f$,

(b) $|H|<|\operatorname{supp} f|+\delta$,

(c) If $x \notin H$ and $y \in \bigcup_{K \in \mathcal{K}} K$ then $|x-y|>\eta$,

(d) If $K \in \mathcal{K}$ then $10^{2} \eta^{-1} \int_{K} f(t) d t<\delta$.

Then, given any $K_{1} \in \mathcal{K}$ and any $\epsilon>0$, we can find a positive infinitely differentiable function $F$ such that

$(d)^{\prime}$ If $K \in \mathcal{K}$ then $10^{2} \eta^{-1} \int_{K} F(t) d t<\delta$,

and

(i) $\hat{F}(0)=1$,

(ii) $|\hat{F}(u)-\hat{f}(u)| \leq \epsilon$ if $|\hat{f}(u)|>\delta / 4$,

(ii) $|\hat{F}(u)| \leq \delta / 2$ if $|\hat{f}(u)| \leq \delta / 4$,

(iii) $\left|S_{n}(F, x)\right| \leq\left|S_{n}(f, x)\right|+\epsilon$ for all $n \geq 0$ and all $x \notin H$ with $\left|S_{n}(f, x)\right|>\delta / 4$,

(iii) $\left|S_{n}(F, x)\right| \leq \delta / 2$ for all $n \geq 0$ and all $x \notin H$ with $\left|S_{n}(f, x)\right| \leq \delta / 4$,

(iv) We can find a finite set $\mathcal{I}_{K_{1}}$ of closed intervals such that $\bigcup_{I \in \mathcal{I}_{K_{1}}} I \supseteq \operatorname{supp} F \cap K_{1}$ but $\sum_{I \in \mathcal{I}} h(|I|) \leq \epsilon$,

(v) $\operatorname{supp} F \subseteq \operatorname{supp} f$.

Proof of Lemma 9. First apply Lemma 10 to obtain $\mathcal{K}$ with the properties given in that Lemma. We suppose that $\mathcal{K}$ contains $M$ intervals $K(1), K(2), \ldots, K(M)$. Now set 
$\epsilon=\delta /(4 M)$, and $F_{0}=f$. By applying Lemma 11 repeatedly we obtain a sequence of $M$ positive infinitely differentiable functions $F_{j}$ with $\hat{F}_{j}(0)=1$ such that if $1 \leq j \leq M$.

(i) $\hat{F}_{j}(0)=1$,

(ii) $j\left|\hat{F}_{j}(u)-\hat{F}_{j-1}(u)\right| \leq \epsilon$ if $\left|\hat{F}_{j-1}(u)\right|>\delta / 4$,

(ii) ${ }_{j}^{\prime}\left|\hat{F}_{j}(u)\right| \leq \delta / 2$ if $\left|\hat{F}_{j-1}(u)\right| \leq \delta / 4$,

(iii) $)_{j}\left|S_{n}\left(F_{j}, x\right)\right| \leq\left|S_{n}\left(F_{j-1}, x\right)\right|+\epsilon$ for all $n \geq 0$ and all $x \notin H$ with $\left|S_{n}\left(F_{j-1}, x\right)\right|>$ $\delta / 4$

(iii) ${ }_{j}^{\prime}\left|S_{n}\left(F_{j}, x\right)\right| \leq \delta / 2$ for all $n \geq 0$ and all $x \notin H$ with $\left|S_{n}\left(F_{j-1}, x\right)\right| \leq \delta / 4$,

(iv) ${ }_{j}$ We can find a finite set $\mathcal{I}_{K(j)}$ of closed intervals such that $\bigcup_{I \in \mathcal{I}_{K(j)}} I \supseteq \operatorname{supp} F \cap$ $K(j)$ but $\sum_{I \in \mathcal{I}} h(|I|) \leq \epsilon$,

$(\mathrm{v})_{j} \operatorname{supp} F_{j} \subseteq \operatorname{supp} F_{j-1}$.

If we now set $F=F_{M}$ and $\mathcal{I}=\bigcup_{j=1}^{M} \mathcal{I}_{K(j)}$ then the conclusions of Lemma 9 can be read off.

Proof of Lemma 11. We may suppose $\epsilon<10^{-2} \delta$ and $\eta<1$. Choose positive infinitely differentiable functions $\phi_{1}$ and $\phi_{2}$ such that $\phi_{1}+\phi_{2}=1, \phi_{1}(t)=0$ for all $t \notin K_{1}$ and we can find two intervals $J_{1}$ and $J_{2}$ such that

$$
h\left(\left|J_{1}\right|\right)+h\left(\left|J_{2}\right|\right) \leq \epsilon / 2 \text {, and } \phi_{2}(t)=0 \text { for all } t \in K_{1} \backslash\left(J_{1} \cup J_{2}\right) .
$$

We set $f_{1}=\phi_{1} f, f_{2}=\phi_{2} f$.

Since $f_{1}$ and $f_{2}$ are infinitely differentiable, we can find a positive integer $N$ such that

$$
\sum_{|u| \geq N}\left|\hat{f}_{1}(u)\right|+\left|\hat{f}_{2}(u)\right| \leq \delta / 16 .
$$

We now approximate $f_{1}$ in the weak star sense by a function $g_{1}$ of the form

$$
g_{1}=\sum_{j=1}^{M} \lambda_{j} \delta_{x_{j}} * G
$$

where $\delta_{x_{j}}$ is the Dirac delta measure at $x_{j}, G$ is an infinitely differentiable function of integral 1 and support a small interval containing 0 . Provided we take $M$ large enough and the support of $G$ small enough and choose the $\lambda_{j}$ and $x_{j}$ appropriately, we can ensure that

$$
\sum_{|u| \leq N}\left|\hat{f}_{1}(u)-\hat{g}_{1}(u)\right| \leq \epsilon / 16
$$

supp $g_{1} \subseteq K_{1}$, and in addition

(i) ${ }^{\prime} \hat{g}_{1}(0)=\hat{f}_{1}(0)$,

(iv $)^{\prime}$ We can find a finite set of closed intervals $\mathcal{J}$ such that $\bigcup_{J \in \mathcal{J}} I \supseteq \operatorname{supp} g_{1}$ but $\sum_{J \in \mathcal{J}} h(|J|) \leq \epsilon / 2$,

$(\mathrm{v})^{\prime} \operatorname{supp} g_{1} \subseteq \operatorname{supp} f_{1}$.

Now set $F=g_{1}+f_{1}$ and $\mathcal{I}_{K_{1}}=\mathcal{J} \cup\left\{J_{1}\right\} \cup\left\{J_{2}\right\}$. Conclusions (d)' and (i) follow from $(\mathrm{i})^{\prime}$ and the definitions of $f_{1}$ and $f_{2}$. Similarly conclusions (iv) and (v) follow more or less directly from (iv)' and (v)'. To prove (ii) observe that, if $|\hat{f}(u)|>\delta / 4$, then (since 
$\left.\left|\hat{f}_{1}(u)\right|+\left|\hat{f}_{2}(u)\right| \geq|\hat{f}(u)|\right)$ equation (1) tells us that $|u|<N$ and so equation (2) gives us

$$
|\hat{F}(u)-\hat{f}(u)|=\left|\hat{g}_{1}(u)-\hat{f}_{1}(u)\right| \leq \epsilon .
$$

To prove (ii)' observe that

$$
\left\|f_{1}\right\|_{1}+\left\|g_{1}\right\|_{1}=\hat{f}_{1}(0)+\hat{g}_{0}(0)=2 \hat{f}_{1}(0) \leq 10^{-2} \eta^{-1} \delta<\delta / 4
$$

by condition (d) and so

$$
|\hat{F}(u)| \leq|\hat{f}(u)|+\left|\hat{g}_{1}(u)-\hat{f}_{1}(u)\right| \leq|\hat{f}(u)|+\left|\hat{g}_{1}(u)\right|+\left|\hat{f}_{1}(u)\right| \leq|\hat{f}(u)|+\delta / 4
$$

for all $u$.

The proof of (iii) and (iii)' follows the same pattern but constitutes a central step in the proof. Suppose $x \notin H$ and so in particular, $x \notin \operatorname{supp} f$. Since $f$ is smooth, $\sum_{r=-\infty}^{\infty} \hat{f}(r) \exp$ ir $x$ converges absolutely to $f(x)=0$ and so, if $\left|S_{n}(f, x)\right|>\delta / 4$, equation (1) tells us that $|u|<N$. Equation (2) now tells us that

$$
\left|S_{n}(F, x)-S_{n}(f, x)\right|=\left|S_{n}\left(g_{1}, x\right)-S_{n}\left(f_{1}, x\right)\right| \leq \sum_{|u| \leq N}\left|\hat{f}_{1}(u)-\hat{g}_{1}(u)\right| \leq \epsilon
$$

and so

$$
\left|S_{n}(F, x)\right| \leq\left|S_{n}(f, x)\right|+\epsilon
$$

as required. To prove (iii) ${ }^{\prime}$, observe that, as we noted in the previous paragraph $\left\|f_{1}\right\|+$ $\left\|g_{1}\right\| \leq 10^{-2} \eta^{-1} \delta$ and, using condition (c), $|x-y|>\eta$. Now, using the Dirichlet formula

$$
S_{n}(\mu, x)=\int \frac{\sin \left(n+\frac{1}{2}\right)}{\sin \frac{t}{2}} d \mu(t)
$$

we have

$$
\left|S_{n}\left(f_{1}, x\right)\right| \leq \frac{\pi}{\eta}\left\|f_{1}\right\|, S_{n}\left(g_{1}, x\right) \mid \leq \frac{\pi}{\eta}\left\|g_{1}\right\|
$$

and

$$
\begin{aligned}
\left|S_{n}(F, x)\right| & \leq\left|S_{n}(f, x)\right|+\left|S_{n}\left(g_{1}, x\right)-S_{n}\left(f_{1}, x\right)\right| \\
& \leq\left|S_{n}(f, x)\right|+\left|S_{n}\left(g_{1}, x\right)\right|+\left|S_{n}\left(f_{1}, x\right)\right| \leq\left|S_{n}(f, x)\right|+\delta / 4
\end{aligned}
$$

This completes the proof of the main lemma and so of Theorem 4 .

5. Algebraic independence. We now turn to the proof of the full Theorem 2. To this end we use the following modification of Lemma 5.

LEMma 12. We can find a sequence $K_{j}$ with the following property. Given any strictly increasing continuous function $h:[0, \infty) \rightarrow[0, \infty)$ with $h(0)=0$ and any sequence $\epsilon_{j}$ with $\epsilon_{j}>0$ we can find a sequence of positive measures $\mu_{j}$ and Borel sets $G_{j}$ such that

(i) $\hat{\mu}_{j}(0)=1$,

(ii) $)_{j} \hat{\mu}_{j}(n) \rightarrow 0$ as $|n| \rightarrow \infty$,

(iii) $)_{j}\left|\hat{\mu}_{j}(u)\right| \leq \epsilon$ for all $u \neq 0$,

(iv) $)_{j}\left|S_{n}\left(\mu_{j}, x\right)\right| \leq K_{j}$ for all $n \geq 0$ and all $x \notin G_{j}$,

(v) $)_{j}\left|G_{j}\right|<2^{-j}$,

(vi $)_{j}$ supp $\mu_{j}$ has Hausdorff $h$ measure zero,

(vii) $\bigcup_{j=1}^{\infty} G_{j}$ independent. 
The proof of Theorem 2 follows that of Theorem 4 . We take

$$
\mu=\sum_{j=1} Q_{j} \mu_{j}
$$

for appropriate trigonometric polynomials $Q_{j}$ and an appropriate $\epsilon_{j}$.

To obtain Lemma 12 we need a more complicated version of Lemma 7 . If $\mathbf{a}=$ $\left(a_{1}, a_{2}, \ldots, a_{M}\right)$ is a non-zero finite sequence of integers and $\delta>0$, let us say that a set $E$ is $(\mathbf{a}, \delta)$ independent if there is no solution of

$$
\sum_{m=1}^{M} a_{m} x_{m}=0
$$

with $x_{m} \in E$ for $1 \leq m \leq M$ and $\min _{m \neq k}\left|x_{m}-x_{k}\right|>\delta$. We choose, once and for all, a sequence $\mathbf{a}(n)$ of non-zero finite sequences such that each possible sequence appears infinitely often.

LEMma 13. We can find a sequence $K_{j}$ with the following property. Given any strictly increasing continuous function $h:[0, \infty) \rightarrow[0, \infty)$ with $h(0)=0$ and any sequence $\epsilon_{j}$ with $\epsilon_{j}>0$ we can find positive infinitely differentiable functions $f_{j, k}$ and sets $G_{j, k}$ such that

$(i)_{k} \hat{f}_{j, k}(0)=1$ for $1 \leq j \leq k$,

(ii) $)_{k}\left|\hat{f}_{j, k}(u)-\hat{f}_{j, k-1}(u)\right| \leq 2^{-k}$ for all $u$ if $1 \leq j \leq k-1$,

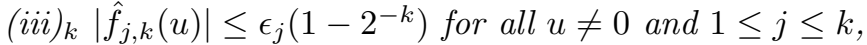

$(\text { iv })_{k}\left|S_{n}\left(f_{j, k}, x\right)\right| \leq K_{j}\left(1-2^{-k}\right)$ for all $n \geq 0$, all $x \notin G_{j, k}$ and $1 \leq j \leq k$,

$(v)_{k}\left|G_{j, k}\right|<2^{-j}\left(1-2^{-k)}\right)$ for all $1 \leq j \leq k$,

$(\text { vi })_{k}$ If $1 \leq j \leq k-1$ we can find a finite set $\mathcal{I}(j, k)$ of closed intervals such that $\bigcup_{I \in \mathcal{I}(j, k)} \supseteq \operatorname{supp} f_{k}$ but $\sum_{I \in \mathcal{I}(j, k)} h(|I|) \leq 2^{-k}$,

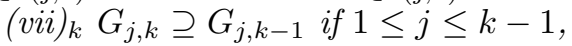

$(\text { viii })_{k} \operatorname{supp} f_{j, k-1} \supseteq \operatorname{supp} f_{j, k}$ if $1 \leq j \leq k-1$,

$(i x)_{k} G_{j, k} \supseteq \operatorname{supp} f_{j, k}$ for $1 \leq j \leq k$,

$(x)_{k} \bigcup_{j=1}^{k-1} G_{j, k}$ is $\left(\mathbf{a}_{n}, 2^{-k}\right)$ independent.

Proof of Lemma 12 from Lemma 13. This follows the proof of Lemma 5 closely. Using conditions (i) $)_{k}$, (ii) $)_{k}$ and (ix) $)_{k}$ we see that $f_{j, k} m$ converges weakly to a positive measure $\mu_{j}$ with support lying within $G_{j}=\bigcap_{j=k}^{\infty} G_{j, k}$. Conditions (i) $)_{j}$ to $(\mathrm{vi})_{j}$ are deduced in the same way that we obtained conditions (i) to (vi) of Lemma 5.

Suppose now that $\mathbf{a}=\left(a_{1}, a_{2}, \ldots, a_{M}\right)$ is a non-zero finite sequence of integers and $x_{1}, x_{2}, \ldots, x_{M}$ are distinct points of $\bigcup_{j=1}^{\infty}$ supp $\mu_{j}$. Simple arguments show that we can find an $N$ such that

(a) $\max _{m \neq q}\left|x_{m}-x_{q}\right|>2^{-N}$,

(b) $x_{m} \in \operatorname{supp} \bigcup_{j=1}^{N-1} G_{j}$ for all $1 \leq m \leq M$,

(c) $\mathbf{a}_{N}=\mathbf{a}$.

Since $G_{j} \subseteq G_{j, N}$ for each $1 \leq j \leq N$ condition $(\mathrm{x})_{N}$ tells us that

$$
\sum_{m=1}^{M} a_{m} x_{m} \neq 0 .
$$

Thus $\bigcup_{j=1}^{\infty} G_{j}$ is independent and we are done. 
6. Discussion of the inductive step. If we look at Lemma 13 and compare it with Lemma 7 it is clear that (if we proceed as before) each inductive step will itself involve many substeps. The introduction of the function $f_{k, k}$ and the set $G_{k, k}$ will follow Lemma 8. The steps required to ensure (vi) $k$ will follow the method given in Lemma 9. We shall therefore only deal with the steps required to obtain $(\mathrm{x})_{k}$.

Essentially, we need to prove the following version of Lemma 9:

Lemma 14. Suppose that we are given $R$ positive infinitely differentiable functions $f_{k}$ with $\hat{f}_{k}(0)=1[1 \leq k \leq R]$. Then, given any $\delta>0$ and any non-zero finite sequence $\mathbf{a}=\left(a_{1}, a_{2}, \ldots, a_{M}\right)$ of integers, we can find positive infinitely differentiable functions $G_{j}$ and a closed set $H$ such that

(i) $\hat{G}_{k}(0)=1$ for all $1 \leq k \leq R$,

(ii) $\left|\hat{G}_{k}(u)-\hat{f}_{k}(u)\right| \leq \delta$ for all $1 \leq k \leq R$,

(iii) $\left|S_{n}\left(G_{k}, x\right)\right| \leq\left|S_{n}\left(f_{k}, x\right)\right|+\delta$ for all $n \geq 0$, all $1 \leq k \leq R$ and all $x \notin H$,

(iv) $\bigcup_{k=1}^{R} \operatorname{supp} G_{k}$ is $(\mathbf{a}, \delta)$ independent.

(v) $\operatorname{supp} G_{k} \subseteq \operatorname{supp} f_{k}$ for all $1 \leq k \leq R$,

(vi) $H \supseteq \bigcup_{k=1}^{R} \operatorname{supp} f_{k}$,

(vii) $|H|<\left|\bigcup_{k=1}^{R} \operatorname{supp} G_{k}\right|+\delta$.

The rest of this paper is devoted to proving this result.

We shall follow the proof of Lemma 9 with appropriate modifications. As might be expected, we start with a version of Lemma 10.

LEMma 15. Suppose that we are given $R$ positive infinitely differentiable functions $f_{k}$ with $\hat{f}_{k}(0)=1[1 \leq k \leq R]$. Then given any $\delta>0$ and any non-zero finite sequence $\mathbf{a}=\left(a_{1}, a_{2}, \ldots, a_{M}\right)$ of integers we can find an $\eta>0$, a finite collection $\mathcal{K}$ of closed intervals such that every pair of intervals in $\mathcal{K}$ intersect at at most one point, and a closed set $H$ such that

(a) $H \supseteq \operatorname{supp} f$,

(b) $|H|<|\operatorname{supp} f|+\delta$,

(c) If $x \notin H$ and $y \in \bigcup_{K \in \mathcal{K}} K$ then $|x-y|>\eta$,

(d) If $K \in \mathcal{K}$ then $10^{3} \eta^{-1} \int_{K} f_{k}(t) d t<\delta / M$,

(e) If $K \in \mathcal{K}$ then $|K|<\delta / 10$.

Proof. Essentially the same as that of Lemma 10.

Our central step corresponds to Lemma 11.

Lemma 16. Suppose that we are given $R$ positive infinitely differentiable functions $f_{k}$ with $\hat{f}_{k}(0)=1[1 \leq k \leq R]$. together with $\delta>0$, a non-zero finite sequence $\mathbf{a}=$ $\left(a_{1}, a_{2}, \ldots, a_{M}\right)$ of integers, an $\eta>0$, and a finite collection $\mathcal{K}$ of closed intervals such that every pair of intervals in $\mathcal{K}$ intersect at at most one point, and a closed set $H$ such that

(a) $H \supseteq \operatorname{supp} f$,

(b) $|H|<|\operatorname{supp} f|+\delta$,

(c) If $x \notin H$ and $y \in \bigcup_{K \in \mathcal{K}} K$ then $|x-y|>\eta$,

(d) If $K \in \mathcal{K}$ then $10^{3} \eta^{-1} \int_{K} f_{k}(t) d t<\delta / M$. 
(e) If $K \in \mathcal{K}$ then $|K|<\delta / 10$.

Then given any $K_{1}, K_{2}, \ldots, K_{M} \in \mathcal{K}$ such that

$$
\inf _{x \in K_{r}, y \in K_{s}}|x-y|>\delta / 2 \text { whenever } 1 \leq r<s \leq M
$$

and any $\epsilon>0$ we can find $R$ positive infinitely differentiable functions $F_{k}[1 \leq k \leq R]$ such that

$(d)^{\prime}$ If $K \in \mathcal{K}$ then $10^{2} \eta^{-1} \int_{K} F_{k}(t) d t<\delta$, for all $1 \leq k \leq R$

and

(i) $\hat{F}_{k}(0)=1$,

(ii) $\left|\hat{F}_{k}(u)-\hat{f}_{k}(u)\right| \leq \epsilon$ if $\left|\hat{f}_{k}(u)\right|>\delta / 4$,

(ii) $\left|\hat{F}_{k}(u)\right| \leq \delta / 2$ if $\left|\hat{f}_{k}(u)\right| \leq \delta / 4$,

(iii) $\left|S_{n}\left(F_{k}, x\right)\right| \leq\left|S_{n}\left(f_{k}, x\right)\right|+\epsilon$ for all $n \geq 0$ and all $x \notin H$ with $\left|S_{n}\left(f_{k}, x\right)\right|>\delta / 4$,

(iii)' $\left|S_{n}\left(F_{k}, x\right)\right| \leq \delta / 2$ for all $n \geq 0$ and all $x \notin H$ with $\left|S_{n}\left(f_{k}, x\right)\right| \leq \delta / 4$,

(iv) If $x_{m} \in \bigcup_{k=1}^{R} \operatorname{supp} F_{k} \cap K_{m}$ for $1 \leq m \leq M$ then $\sum_{j=1}^{M} a_{j} x_{j} \neq 0$.

(v) $\operatorname{supp} F_{k} \subseteq \operatorname{supp} f_{k}$.

Sketch proof of Lemma 14. This follows the proof of Lemma 9 but the details, if we were to give them, would require quite complex notation. We first need to apply Lemma 15 to obtain $\mathcal{K}$ with the properties given in that Lemma. We now apply Lemma 16 to each possible collection $K_{1}, K_{2}, \ldots, K_{M} \in \mathcal{K}$ such that

$$
\inf _{x \in K_{r}, y \in K_{s}}|x-y|>\delta / 2 \text { whenever } 1 \leq r<s \leq R,
$$

in turn. The arguments of the proof of Lemma 9 from Lemma 11 give, mutatis mutandis, all the conditions of Lemma 14 with the exception of (iv).

To prove (iv), observe that if $x_{m} \in \bigcup_{k=1}^{R} \operatorname{supp} G_{k}$ for $1 \leq m \leq M$ and $\min _{m \neq k}\left|x_{m}-x_{k}\right|>\delta$ then, automatically $x_{m} \in \bigcup_{K \in \mathcal{K}} K$ and there must be a collection $K_{1}, K_{2}, \ldots, K_{M} \in \mathcal{K}$ such that

$$
\inf _{x \in K_{r}, y \in K_{s}}|x-y|>\delta / 2 \text { whenever } 1 \leq r<s \leq R
$$

and $x_{m} \in K_{m}$ for $1 \leq m \leq M$. Thus, by looking at condition (vi) of Lemma 16, for the appropriate step we obtain

$$
\sum_{j=1}^{m} a_{j} x_{j} \neq 0
$$

as required.

Sketch proof of Lemma 16. We may suppose $\epsilon<10^{-2} \delta$ and $\eta<1$. Choose positive infinitely differentiable functions $\phi_{1}$ and $\phi_{2}$ such that $\phi_{1}+\phi_{2}=1, \phi_{1}(t)=1$ for all $t \in$ and $\phi_{1}(t)=0$ unless $t$ lies in some interval $K \in \mathcal{K}$ which shares an end point with one of the $K_{m}$ with $1 \leq m \leq M$. We set $f_{1, r}=\phi_{1} f_{r}, f_{2}=\phi_{2} f_{r}$.

Since $f_{1, r}$ and $f_{2, r}$ are infinitely differentiable we can find a positive integer $N$ such that

$$
\sum_{|u| \geq N}\left|\hat{f}_{1}(u)\right|+\left|\hat{f}_{2}(u)\right| \leq \delta / 16
$$


for all $1 \leq r \leq R$. We now approximate the $f_{1, r}$ in the weak star sense by a function $g_{1, r}$ of the form

$$
g_{1, r}=\sum_{j=1}^{J} \lambda_{j} \delta_{y_{j}} * G
$$

where $\delta_{y_{j}}$ is the Dirac delta measure at $y_{j}$ and $G$ is an infinitely differentiable function of integral 1 and support a small interval containing 0 . We demand that the $y_{j}$ be independent.

Provided we take $M$ large enough and choose the $\lambda_{j}$ and $x_{j}$ appropriately we can ensure that, provided only $\operatorname{supp} G$ lies within a sufficiently small distance of 0 ,

$$
\sum_{|u| \leq N}\left|\hat{f}_{1, r}(u)-\hat{g}_{1, r}(u)\right| \leq \epsilon / 16
$$

and in addition

(i) $\hat{g}_{1, r}(0)=\hat{f}_{1}(0)$,

$(\mathrm{v})^{\prime} \operatorname{supp} g_{1, r} \subseteq \operatorname{supp} f_{1, r}$,

$(\mathrm{d})^{\prime \prime} \int_{K} g_{1, r}(t) d t=\int_{K} f_{1, r}(t) d t$ for all $K \in \mathcal{K}$.

Since the $y_{j}$ are independent we can find a $\rho>0$ such that, if $\left|x_{j}-y_{j}\right| \leq \rho$ for $1 \leq j \leq M$

$$
\sum_{j=1}^{M} a_{j} x_{j} \neq 0 .
$$

Choose $G$ so that supp $G \subseteq[-\omega, \omega]$ and all the conclusions of the previous paragraph hold. Conclusion (iv) of our lemma is now immediate and conclusion (d) follows from (d) ${ }^{\prime \prime}$. The remaining conclusions are proved in the same way as the corresponding conclusions in Lemma 11.

It would be nice to think that the correction theorems obtained in this paper had something to do with questions of pointwise convergence such as are considered in Carleson's theorem, but, so far as I can see, they do not. It would be surprising if they did, since the methods of this paper may be complicated but are certainly not deep.

\section{References}

[1] T. W. Körner, J. London Math. Soc. (2) 60 (1999), 548-560. 